\title{
Origin of the subscapular artery in the South African Black population
}

\author{
N.K. Xhakaza ${ }^{1}$, K.S. Satyapal ${ }^{2}$ \\ ${ }^{1}$ Department of Human Anatomy and Physiology, Faculty of Health Sciences, Doornfontein Campus, \\ University of Johannesburg, Johannesburg, South Africa \\ ${ }^{2}$ Department of Clinical Anatomy, School of Laboratory Medicine and Medical Sciences, Westville Campus, \\ College of Health Sciences, University of KwaZulu-Natal, Durban, South Africa
}

[Received 17 December 2013; Accepted 28 March 2014]

There is great variability in the branching patterns of the axillary artery (AA). Racial differences have been reported to play a role in this variability. The subscapular artery (SA) is the largest and most variable branch of the AA. It usually arises from the third part of the AA at the inferior border of the subscapularis muscle. Approximately $4 \mathrm{~cm}$ from its origin, the SA divides into the circumflex scapular and thoracodorsal arteries. Two types of the SA have been described, depending on the site of its origin from the $A A$ in relation to the point where the AA passes between the lateral and medial roots of the median nerve. It is referred to as the superficial SA (SSA), when proximal, and the deep SA (DSA), when distal to this point, respectively. This study aimed to determine the site and distance of origin of the SA from the outer border of the first rib in the South African Black population. The study comprised bilateral gross anatomical dissections of 50 adult Black South African cadavers ( $n=100 \mathrm{AAs}$ ). The site and distance of origin of each vessel from the outer border of the first rib were recorded. Additional branches and variations were also noted. The SSA and DSA were found in $52.8 \%$ and $47.2 \%$ of cases, respectively. In $16.8 \%$ of cases, the SA gave rise to the posterior circumflex humeral artery and the lateral thoracic artery in 33.7\% cases. The SA was absent in $11 \%$ of the cases. The prevalence of the SSA reported in this study differs from values ranging between $1.7 \%$ and $16 \%$ reported in the literature. The high incidence of the SSA in this study may have clinical significance as a superficial course of the arteries make them vulnerable to injury during surgical procedures. (Folia Morphol 2014; 73, 4: 486-491)

Key words: axillary artery, subscapular artery, superficial subscapular artery, deep subscapular artery

\section{INTRODUCTION}

The subscapular artery (SA) is the largest branch of the axillary artery (AA) [17]. It usually arises from the third part of the $A A$ at the inferior border of the subscapularis muscle, which it follows to the inferior angle of the scapula where it anastomoses with the lateral thoracic and intercostal arteries and the deep branch of the transverse cervical artery [17]. It is accompanied distally by the thoracodorsal nerve and supplies adjacent muscles and the thoracic wall. Approximately $4 \mathrm{~cm}$ from its origin, the SA divides into the circumflex scapular and thoracodorsal arte-

Address for correspondence: Prof. K.S. Satyapal, Department of Clinical Anatomy, School of Laboratory Medicine and Medical Sciences, College of Health Sciences, University of KwaZulu-Natal, Private Bag X54001, Durban, 4000, South Africa, tel:+ 27312607110 , fax:+ 27312607890 , e-mail: satyapalk@ukzn.ac.za 
Table 1. Comparison of sites and modes of origin of subscapular artery

\begin{tabular}{llcc}
\hline Author (year) & Variation of origin & Sample no. & Incidence \\
\hline DeGaris and Swartley (1928) & Second part of axillary artery & 512 & $5.1 \%$ \\
& Third part of axillary artery & $94.1 \%$ \\
\hline Huelke (1958) & First part of axillary artery & $0.6 \%$ \\
& Second part of axillary artery & $15.7 \%$ \\
\hline Lippert and Pabst (1985) & Common trunk with posterior circumflex humeral artery & Not recorded & $20 \%$ \\
& from axillary artery & Not recorded & $10 \%$ \\
\hline Patnaik et al. (2000) & Common trunk with lateral thoracic artery from axillary artery & 50 & $16 \%$ \\
& Second part of axillary artery & $80 \%$ \\
\hline Saeed et al. (2003) & Third part of axillary artery & 106 & $3.8 \%$ \\
\hline Huelke (2005) & Common trunk with posterior circumflex humeral artery & 178 & $15.7 \%$ \\
\hline Bergman et al. (2006) & Second part of axillary artery & Not recorded & $15 \%$ \\
& Second part of axillary artery & Not recorded & $6 \%$ \\
\hline Karambelkar et al. (2011) & Common trunk with transverse cervical artery & & $1.66 \%$ \\
\hline Gaur et al. (2012) & from thyrocervical trunk of subclavian artery & 60 & $8.33 \%$ \\
\hline Present study (2014) & Second part of axillary artery & 40 \\
\hline & Common trunk with posterior circumflex humeral artery & 50 & $4 \%$ \\
\hline
\end{tabular}

ries [17]. Keen [10] and Patnaik et al. [13] reported the mean distance of origin of the SA from the AA as $7.3 \mathrm{~cm}$ and $6.69 \mathrm{~cm}$, respectively. The SA has been described as the most variable branch of the AA [1]. Saralaya et al. [16] reported a case of a large collateral branch viz. the 'subscapular common trunk' which arose from the AA at the medial border of the pectoralis minor muscle. The 'subscapular common trunk' gave rise to many branches which normally arise from the second or third part of the AA. Bhat et al. [3] also reported the same variation of the SA in a South Indian male cadaver. In both cases, the thoracodorsal nerve crossed superficial to the 'subscapular common trunk'. The case of the subscapular common trunk reported by Lee and Kim [11] was unique in that the thoracodorsal artery was a direct branch of the second part of the AA. Ramesh et al. [14] recorded a peculiar case in which the SA, the anterior and posterior circumflex humeral arteries and the profunda brachii artery arose from a common trunk that originated from the third part of AA. Yamada [18] distinguished two types of SA: Type 1 - arose from the AA proximal to the point where the AA passed between the lateral and medial roots of median nerve; Type 2 - arose from the AA distal to this point and is referred to as deep SA (DSA). Type 1 SA crossed over the medial cord of the brachial plexus to reach the upper lateral thoracic wall, gave off the lateral thoracic artery and then ran backward on the serratus anterior muscle to reach the deep region of the axilla. Yamada [18] named this type the superficial SA (SSA). Aizawa et al. [1] extended the classification of the SA into 3 types viz.: S-type, I-type and P-type. The S-type was similar to Yamada's 'SSA'. Both the I-type and the P-type arose from the AA distal to the point where the AA penetrated the medial and lateral roots of the median nerve. The I-type ran posteriorly across the medial side of the radial nerve and later ran posteriorly across the lateral side and branched more proximally than the P-type. The P-type arose from the most distal part of the AA. Both the circumflex scapular and thoracodorsal branches of the P-type ran posterior to the radial nerve. Different sites and modes of origin of the SA have been reported by various authors (Table 1 ).

This study aimed to determine the site and distance of origin of the SA in the South African Black population.

\section{MATERIALS AND METHODS}

This study comprised of 100 AAs ( $n=100$ : 50 right and 50 left) which were obtained from gross anatomical dissections of 12 female and 38 male adult cadaveric specimens of indigenous Black South 
Africans. These specimens were obtained from the following South African universities: Department of Clinical Anatomy, College of Health Sciences, University of KwaZulu Natal (UKZN), (Westville campus) and the Department of Human Anatomy and Physiology, University of Johannesburg (UJ), (Doornfontein campus), in accordance with the National Health Act (No. 61 of 2003). Ethical clearance was obtained from the Biomedical Research Ethics Committee of UKZN (Ethics number: BE159/010) and the Faculty of Health Sciences Academic Ethics Committee of UJ (Ethics number: AEC90/02-2010).

The following branches of the AA were identified and dissected: superior thoracic artery, thoraco-acromial artery, lateral thoracic artery, SA, anterior and posterior circumflex humeral arteries. Additional branches in each part of the AA were also carefully dissected and recorded. The distance of origin of the SA from the AA was measured by placing the suture silk on the AA. The silk was then pinned at the outer border of the first rib and the distal angle of origin of the SA. The suture silk was later removed with the pins and the distance between the pins measured using a $30 \mathrm{~cm}$ plastic rule. These measurements were repeated 3 times and the average recorded as the mean \pm standard deviation. In the analysis of our results with regard to the origin of the $S A$, the specimens with an absent SA were excluded.

$A \chi^{2}$ test was used to test association between the sites of origin of the SA and the sex and side of the body. A $p$ value $\leq 0.05$ was considered a statistically significant difference. All statistical analysis was done using GraphPad Prism 5.

\section{RESULTS}

The SA was present in $89 \%(89 / 100)$ cases and it was absent in $11 \%(11 / 100)$ of cases.

\section{Site, side and sex of origin of the SA}

The SA originated directly from the second part of the AA proximal to the point where the AA passed between the medial and the lateral roots of median nerve in $52.8 \%$, (47/89) of cases (viz. the SSA) (Fig. 1A, B); [side and sex differentiation: left $21 / 44$ and right 26/45; male $35 / 69$ and female $12 / 20$ ]. In $16.9 \%$ $(15 / 89)$ cases, the SSA was bilateral. In $47.2 \%$, (42/89) of cases, the $S A$ originated directly from the third part of the AA (viz. the DSA) (Fig. 2A, B): [side and sex differentiation: left 23/44 and right 19/45; male $32 / 69$ and female 10/20]. There were no statistically
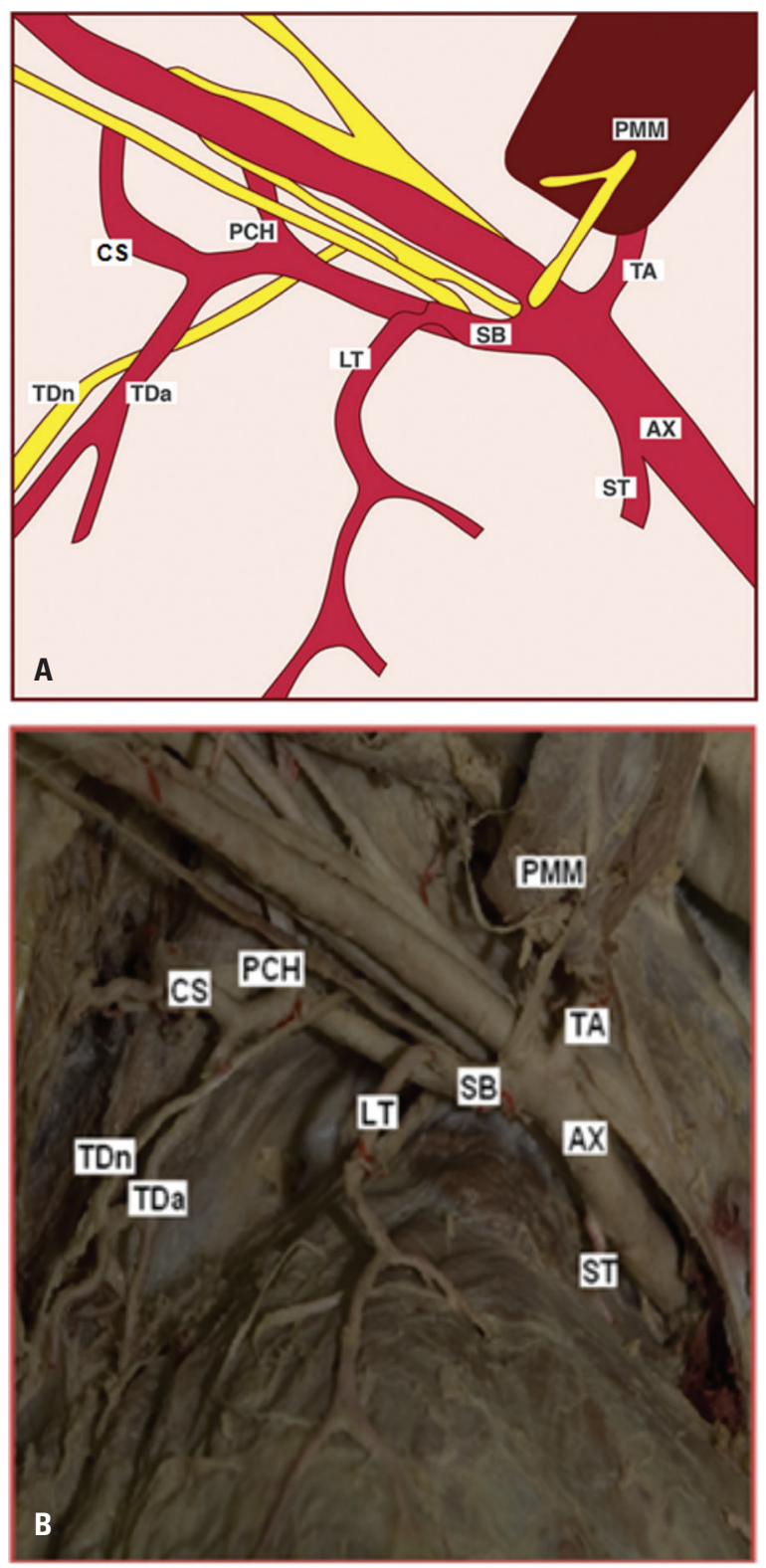

Figure 1. Schematic representation $(\mathbf{A})$ and cadaveric dissection of origin of the subscapular artery from second part of axillary artery (B) (right anterior view); TA — thoraco-acromial artery; PMM — pectoralis minor muscle; AX — axillary artery; SB — subscapular artery; CS - circumflex scapular artery; TDa — thoracodorsal artery; $\mathrm{TDn}$ — thoracodorsal nerve; $\mathrm{LT}$ — lateral thoracic artery; $\mathrm{PCH}$ — posterior circumflex humeral artery; ST — superior thoracic artery.

significant differences when the site of origin of the SA and the side $(p=0.3423)$ and sex $(p=1)$ of specimens were compared (Table 2).

\section{Variations of the subscapular artery}

Branches. When the SA arose from the second part of the AA it branched into the posterior circumflex humeral artery in $16.8 \%$ (15/89) of cases (Fig. 3A, B) 

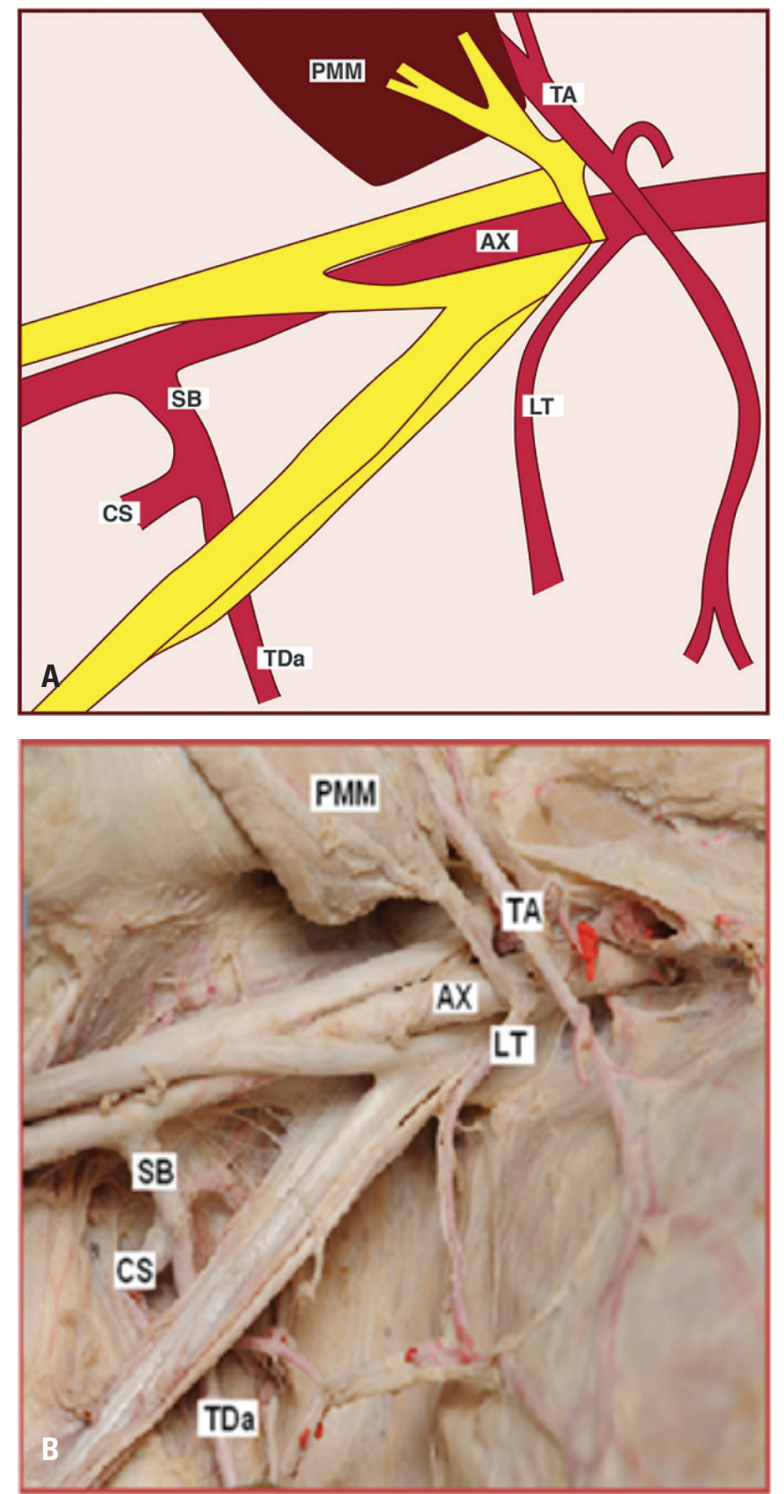

Figure 2. Schematic representation (A) and cadaveric dissection of origin of the subscapular artery from the third part of axillary artery (B) (right anterior view); TA — thoraco-acromial artery, PMM pectoralis minor muscle, AX - axillary artery, SB — subscapular artery, CS - circumflex scapular artery, TDa - thoracodorsal artery, LT — lateral thoracic artery.

and the lateral thoracic artery in $33.7 \%$ of cases (Fig. 4A, B). When the SA arose from the third part of the $A A$, it formed a common trunk with the posterior circumflex humeral artery in $2.2 \%(2 / 89)$ of cases (Fig. 5A, B).
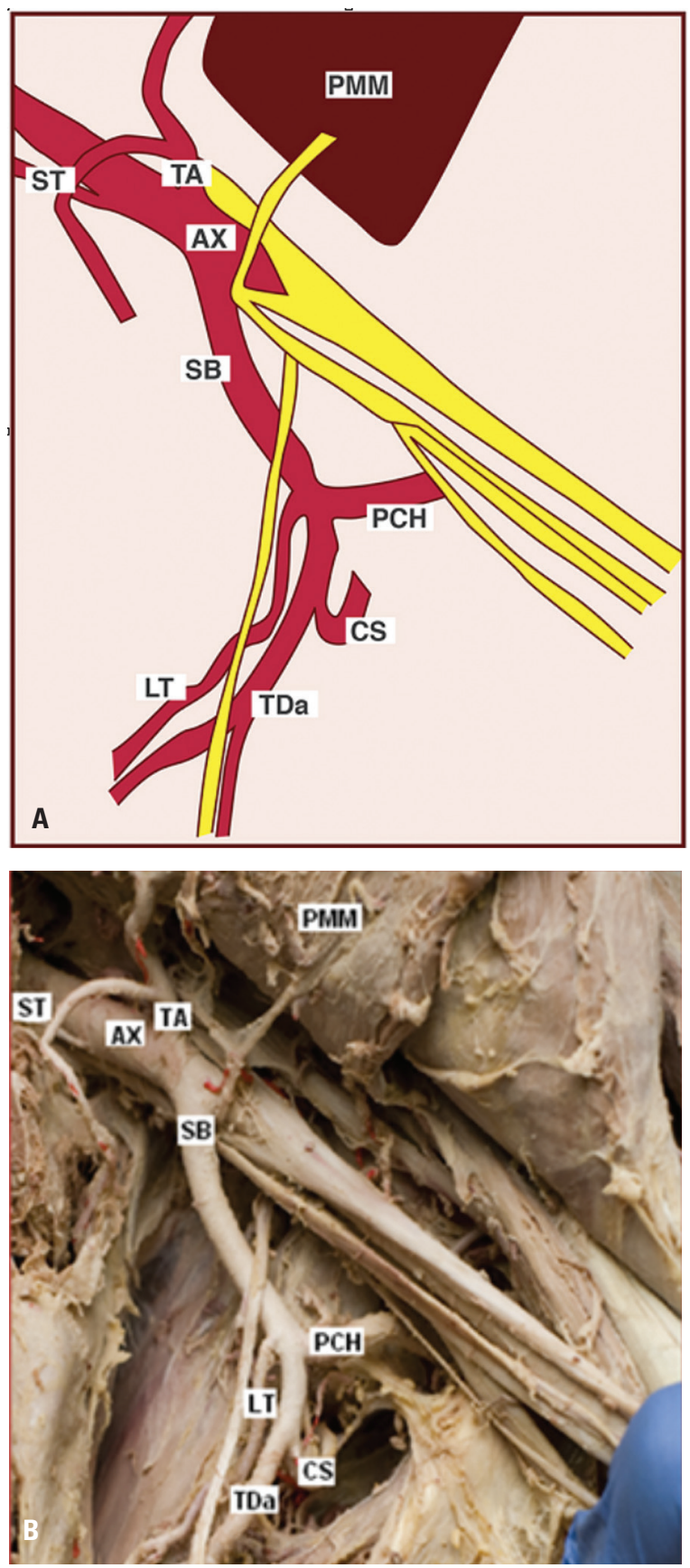

Figure 3. Schematic representation (A) and cadaveric dissection of origin of the posterior circumflex humeral artery from subscapular artery (B) (left anterior view); ST — superior thoracic artery, $\mathrm{TA}$ - thoraco-acromial artery, PMM — pectoralis minor muscle, $\mathrm{AX}$ - axillary artery, $\mathrm{TDa}$ - thoracodorsal artery, $\mathrm{PCH}$ - posterior circumflex humeral artery, LT — lateral thoracic artery, SB - subscapular artery, CS — circumflex scapular artery.

Table 2. Site, side and sex of origin of the subscapular artery

\begin{tabular}{lccccc}
\hline Origin of subscapular artery (SA) & \multicolumn{2}{c}{ Side } & & \multicolumn{2}{c}{ Sex } \\
\cline { 2 - 3 } \cline { 5 - 6 } & Right $(\mathbf{n}=45)$ & Left $(\mathbf{n}=44)$ & & Male $(\mathbf{n}=\mathbf{6 9})$ & Female $(\mathbf{n}=\mathbf{2 0})$ \\
\hline $2^{\text {nd }}$ part of axillary artery (superficial SA) & 26 & 21 & & 35 & 12 \\
$3^{\text {rd }}$ part of axillary artery (deep SA) & 19 & 23 & & 32 & 10 \\
$\mathrm{P}$ & \multicolumn{2}{c}{0.3423} & & & 1 \\
\hline
\end{tabular}



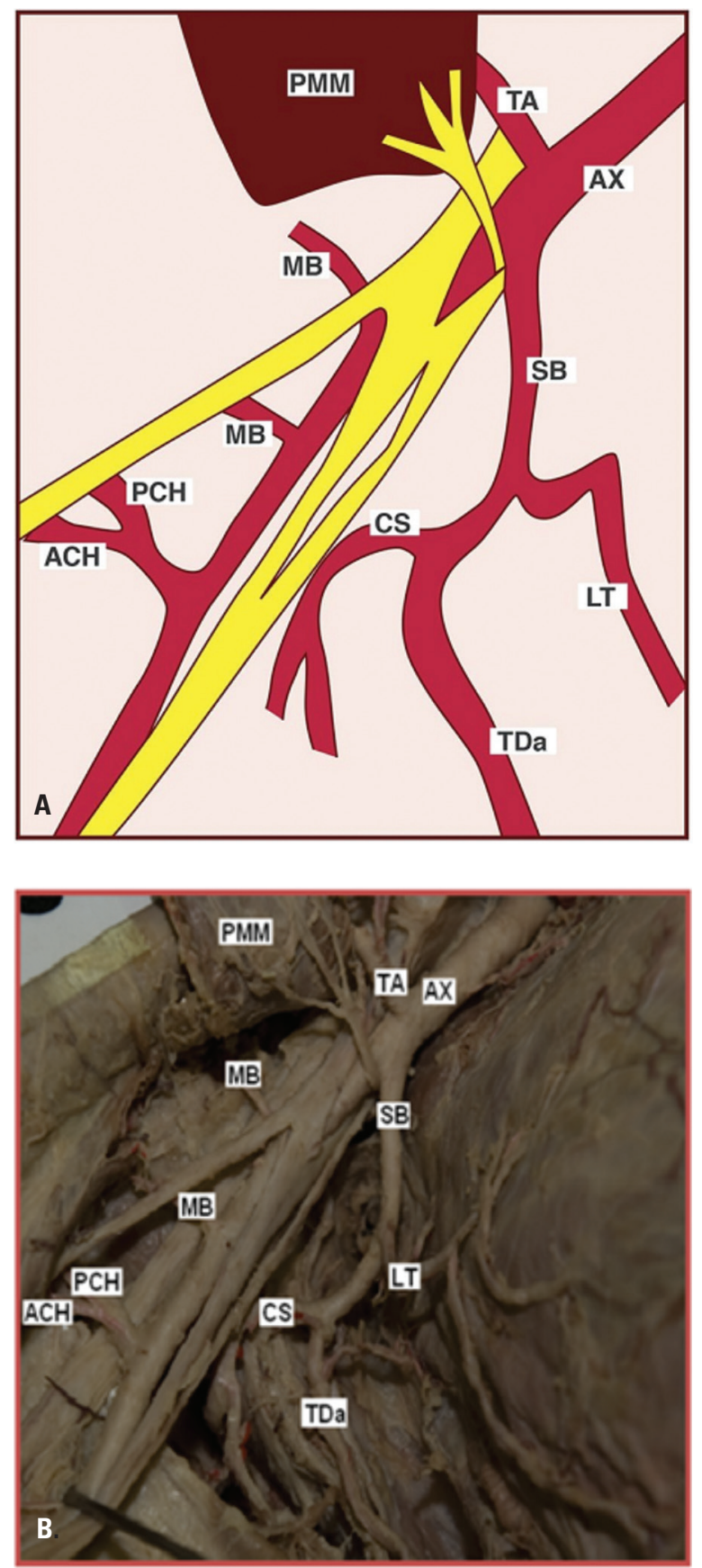

Figure 4. Schematic representation (A) and cadaveric dissection of origin the lateral thoracic artery from the subscapular artery (B) (right anterior view); TA — thoraco-acromial artery, $\mathrm{PMM}$ - pectoralis minor muscle, AX — axillary artery, SB — subscapular artery, CS circumflex scapular artery, TDa - thoracodorsal artery, LT — lateral thoracic artery, ACH - anterior circumflex humeral artery, MB muscular branch, $\mathrm{PCH}$ - posterior circumflex humeral artery.

Absent SA. The SA was absent in 11 cases (11\%), of which 4 were females ( 2 on the left and 2 on the right) and 7 were males ( 4 on the left and 3 on the right). In those 11 cases, the branches of the SA originated as follows: in $54.5 \%,(6 / 11)$
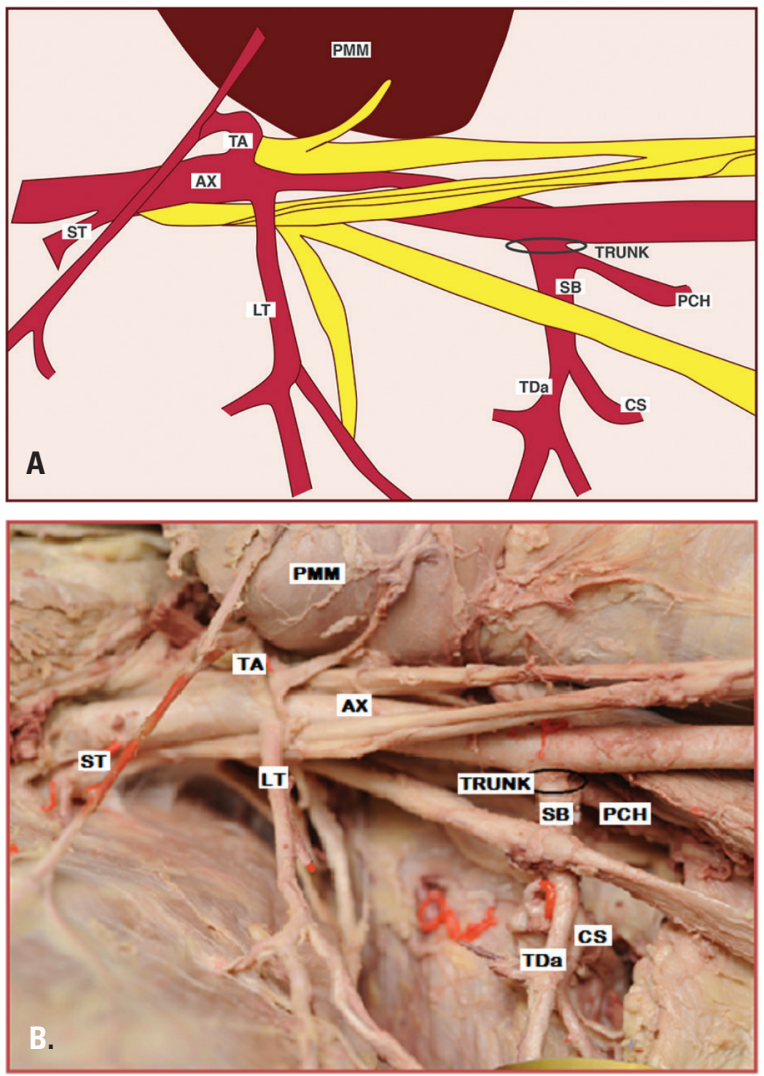

Figure 5. Schematic representation (A) and cadaveric dissection of origin of the posterior circumflex humeral artery from common trunk with subscapular artery off the third part of axillary artery (B) (left anterior view); ST — superior thoracic artery, TA — thoraco-acromial artery, PMM — pectoralis minor muscle, AX — axillary artery, $\mathrm{TDa}$ - thoracodorsal artery, $\mathrm{PCH}$ - posterior circumflex humeral artery, LT — lateral thoracic artery, SB - subscapular artery, CS - circumflex scapular artery, TRUNK - common trunk for posterior circumflex humeral artery and subscapular artery.

of cases, the thoracodrosal artery originated from the $2^{\text {nd }}$ part of the AA and the circumflex scapular artery formed a common trunk with the posterior circumflex humeral artery. In $18.1 \%,(2 / 11)$ of cases, the thoracodorsal artery originated from the $2^{\text {nd }}$ part of the AA and the circumflex scapular artery arose from a common trunk for anterior and posterior circumflex humeral arteries. In $18.1 \%,(2 / 11)$ of cases, the thoracodorsal artery and circumflex scapular artery originated separately from the $2^{\text {nd }}$ part of the AA without forming trunks. In $9 \%(1 / 11)$ of cases, the thoracodorsal artery branched from the $2^{\text {nd }}$ part of the AA and the circumflex scapular artery was a branch of a common trunk for the anterior circumflex humeral artery, posterior circumflex humeral artery and profunda brachii artery. 


\section{Distance of origin of the SA}

The mean distance of origin of the SA from the outer border of the first rib was $61.67 \pm 26.40 \mathrm{~mm}$ on the left and $57.19 \pm 24.76 \mathrm{~mm}$ on the right.

\section{DISCUSSION}

The SA was absent in $11 \%$ of cases in this study which was higher than that reported by Huelke [7] (1.7\%) and Patnaik et al. [13] (4\%).

The SA originated directly from the second part of the AA in $52.8 \%$ cases. This pattern of origin has been referred to as a SSA by Yamada [18] as it originated proximal to the point where the AA passed between the medial and lateral roots of median nerve. This study reports a higher incidence of the SSA than the studies reported by DeGaris and Swartley [5], Patnaik et al. [13], Huelke [8], Bergman et al. [2], Daimi et al. [4], Karambelkar et al. [9] and Gaur et al. [6]. This study observed the highest incidence of origin of the SA (52.8\%) from the second part of the AA as the previous authors reported this incidence to be between $1.7 \%$ and $16 \%$ (Table 1 ).

The origin of the SA from the first part of the AA was not recorded in this study.

When the SA arose from the second part of the $A A$, it branched into the posterior circumflex humeral artery (16.8\%) and the lateral thoracic artery (33.7\%). The latter results compare favourably with that reported by Bhat et al. [3], Lee and Kim [11] and Saralaya et al. [16].

In $47.2 \%$ of cases, the SA originated directly from the $3^{\text {rd }}$ part of the AA. When the SA arose from the $3^{\text {rd }}$ part of the $A A$, it formed a common trunk with the posterior circumflex humeral artery in $2.2 \%$ of cases. These results concur with Lippert and Pabst [12], Saeed et al. [15] and Karambelkar et al. [9].

The mean distance of origin of the SA, as measured from the outer border of the first rib, was reported to be $73 \mathrm{~mm}$ [10] and $66.9 \mathrm{~mm}$ [13]. In the current study, the mean was however found to be $61.67 \mathrm{~mm}$ on the left and $57.19 \mathrm{~mm}$ on the right. The disparity in the results of the current study and those of Keen [10] and Patnaik et al. [13] probably arose from the fact that they pooled left and right measurements.

The SSA [18] may be of clinical importance as arteries that take a more superficial course than expected are more vulnerable to iatrogenic injuries; hence, this study may provide useful clinical information to clinicians working in this area.

\section{CONCLUSIONS}

The most characteristic feature of the AA branching pattern in this study is the high incidence of origin of the SA from the second part of the AA. There is no association between the site of origin of the SA with either the side of the body or the sex of the cadaver.

\section{ACKNOWLEDGEMENTS}

The authors wish to thank Drs J. Naidu and N.O. Ajayi and Ms L. Lazarus and N. Naidoo for their kind assistance.

\section{REFERENCES}

1. Aizawa Y, Ohtsuka K, Kumaki K (1995) Examination on the courses of the arteries in the axillary region I. The course of the subscapular arterial system, especially the relationships between the arteries and the posterior cord of the brachial plexus. Kaibokaku Zasshi, 70: 554-568.

2. Bergman RA, Afifi AK, Miyauchi R (2006) Illustrated Encyclopedia of Human Variation. Bookworm Investigation \& Publishing Consortium, Lowa city.

3. Bhat MR, Gowda S, Potu B, Rao M (2008) A unique branching pattern of axillary artery in a South Indian male cadaver. Bratisl Lek Listy, 109: 587-589.

4. Daimi SR, Siddiqui AU, Wabale RN (2010) Variations in the branching pattern of axillary artery with high origin of radial artery. Int J Anat Variations, 3: 76-77.

5. DeGaris CF, Swartley WB (1928) The axillary artery in white and Negro stocks. Am J Anat, 41: 353-397.

6. Gaur S, Katariya SK, Vaishnani H, Wani IN, Bondre KV, Shah GV (2012) A cadaveric study of branching pattern of the axillary artery. Int J Biol Med Res, 3: 1388-1391.

7. Huelke DF (1958) Variation in origin of branches of the axillary artery. Anat Record, 135: 35-41.

8. Huelke DF (2005) Variation in the origins of the branches of the axillary artery. Anat Record, 135: 33-41.

9. Karambelkar RR, Shewale AD, Umarji BN (2011) Variations in branching pattern of axillary artery and its clinical significance. Anatomica Karnataka, 5: 47-51.

10. Keen JA (1961) A study of arterial variations in the limbs with special reference to symmetry of vascular patterns. Am J Anat; 108: 245-261.

11. Lee JH, Kim DK (2008) Bilateral variations in the origin and the branches of the subscapular artery. Clin Anat, 21: 783-785.

12. Lippert H, Pabst R (1985) Axillary artery. Arterial variations in man. J.F. Bergmann Verlag, Munchen.

13. Patnaik VVG, Kalsey G, Singla Rajan K (2000) Branching pattern of axillary artery: a morphological study. J Anat Soc India, 49: 127-132.

14. Ramesh R, Shetty P, Suresh R (2008) Abnormal branching pattern of the axillary artery and its clinical significance. Int J Morphol, 26: 389-392.

15. Saeed M, Rufai AA, Elsayed SE, Sadiq MS (2002) Variations in the subclavian-axillary arterial system. Saudi Med J, 2: 206-212.

16. Saralaya V, Joy T, Madhyastha S, Vadgaonkar R, Saralaya $S$ (2008) Abnormal branching of the axillary artery: subscapular common trunk. A case report. Int J Morphol, 26: 963-966.

17. Standring S (2008) Gray's anatomy. The anatomical basis of clinical practice. $40^{\text {th }}$ Ed. Elsevier Churchill Livingstone, British library.

18. Yamada M (1967) Significance of the superficial subscapular artery, new designation (in Japanese). Nihon lji Shimpo, 60: 3-7. 